\title{
Evaluating the buffering vs. direct effects hypotheses of emotional social support on inflammatory markers: The Multi-Ethnic Study of Atherosclerosis
}

\author{
Briana Mezuk $^{\mathrm{a}, *}$, Ana V. Diez Roux ${ }^{\mathrm{b}}$, Teresa Seeman ${ }^{\mathrm{c}}$ \\ ${ }^{a}$ Department of Epidemiology and Community Health, Virginia Commonwealth University, USA \\ ${ }^{\mathrm{b}}$ Center for Integrative Approaches to Health Disparities, University of Michigan School of Public Health, USA \\ ' School of Medicine, Division of Geriatrics, University of California, Los Angeles, USA
}

\section{A R T I C L E I N F O}

\section{Article history:}

Received 18 February 2010

Received in revised form 17 May 2010

Accepted 11 June 2010

Available online 25 June 2010

\section{Keywords:}

Social support

Inflammation

Aging

Gender differences

\begin{abstract}
A B S T R A C T
Social support is associated with cardiovascular disease mortality, however, the physiologic mechanisms underlying this relationship remains unspecified. This study evaluated the association of social support with inflammatory markers associated with cardiovascular risk: C-reactive protein (CRP), interleukin-6 (IL-6), and fibrinogen. We evaluated two competing models of the support-inflammation relationship: first, that low social support is directly associated with inflammation, and second, that high support acts to buffer the effect of stress on inflammation. Using data from the baseline interview of the Multi-Ethnic Study of Atherosclerosis ( $N=6814,53 \%$ female, age $45-84$ years) we assessed the independent and interacting associations of social support and stress with inflammation. Social support was measured by the emotional social support index. Stressors in multiple domains (work, family, finances, interpersonal) were assessed. Serum CRP, IL-6, and fibrinogen were analyzed from fasting samples using high-sensitivity assays. Multivariate linear regression, including models stratified by gender and age group (45-64 and 65-84 years), was used to assess the direct and buffering relationships between social support, stress, and inflammation. In bivariate analyses low social support was associated with higher levels of all three markers. In adjusted models, low support was associated with higher $\operatorname{lnCRP}(\mathrm{B}: 0.15,95 \% \mathrm{CI}: 0.01,0.30$ ) among men but not women. High social support buffered the relationship between stress and CRP among middle-aged women only ( $P$ for interaction 0.042 ). Overall, social support was only modestly associated with inflammation in this relatively healthy sample, and these relationships varied by age and gender.
\end{abstract}

(c) 2010 Elsevier Inc. All rights reserved.

\section{Introduction}

Investigations of the interrelationships between the social environment and health have established that low social support, poor social integration and social isolation are associated with increased mortality, particularly from cardiovascular disease (CVD) ${ }^{1}$ (Berkman et al., 2003; Brummett et al., 2001; Frasure-Smith et al., 2000). Despite this epidemiologic evidence, the physiologic mechanisms underlying these relationships remain unspecified (Berkman et al., 2003; Knox and Uvnas-Moberg, 1998). Several researchers have speculated that alterations in immune function may be a mechanism by which psychosocial exposures, including social support,

\footnotetext{
* Corresponding author. Address: Virginia Commonwealth University School of Medicine, Department of Epidemiology and Community Health, P.O. Box 980212, Richmond, VA 23298, USA. Fax: +1 8046282510.

E-mail address: bmezuk@vcu.edu (B. Mezuk).

1 Abbreviations used: BMI: body mass index; CRP: C-reactive protein; CVD: cardiovascular disease; ELISA: enzyme-linked immunosorbant assay; IL-6: interleukin-6; MESA: multi-ethnic study of atherosclerosis; NSAID: non-steroidal antiinflammatory drug; PSS: perceived social support.
}

affect health. In particular, low social support has been associated with elevated levels of circulating inflammatory markers, including cytokines (e.g., interleukin-6 (IL-6), TNF- $\alpha$ ), acute phase proteins (e.g., C-reactive protein), and clotting factors (e.g., fibrinogen) (Seeman et al., 1994; Uchino et al., 1999) that have been implicated in risk of CVD. However, not all studies have reported a significant association between social support and systemic inflammation (McDade et al., 2006); and even in those instances where support is associated with inflammation, it is unclear whether this relationship is due to a direct (i.e., independent) influence on physiology, or if support simply buffers (i.e., moderates) the effect of negative experiences (e.g., daily hassles, caregiving, stressful life events) which have direct effects on physiology (Cohen and Wills, 1985).

An integrative framework of the ways social experiences influence health should address the potential that the relationship between social support and physiologic indicators such as inflammation may vary by age and sex (Seeman and Crimmins, 2001). As social roles evolve over the life course (i.e., marriage, parenthood), the influence of social experiences on health may also change. As with other psychosocial characteristics, social support may have 
cumulative effects over time, as well as acute effects in the context of events that affect social life (i.e., divorce, retirement) (Uchino et al., 1999). Also, many aspects of the receipt and provision of social support vary by sex (i.e., women are more likely to experience widowhood and are more likely to be caretakers than men) (Moen, 2001), and thus the implications for social relations to health may likewise differ for men and women (Ajrouch et al., 2005) Consistent with this general framework, there is suggestive evidence that the physiologic correlates of social integration and support vary by age and gender (Ford et al., 2006; Hughes, 2007). For example, Ford and colleagues (2006) reported that low social integration was associated with elevated C-reactive protein among older men, but not among women or younger men (Ford et al., 2006). Loucks and colleagues (2006a,b) also reported a significant association between CRP levels and social integration among older men but not older women (Loucks et al., 2006a). However, relatively few studies have systematically examined variation by age and gender in this relationship, and most have only examined a single indicator of immune function. Thus, a systematic investigation of whether the relationship between social support and inflammatory indicators of CVD risk varies by age and gender is warranted.

The goal of this paper is to explore the relationship between social support and three markers of inflammation that have been implicated in CVD, interleukin-6, fibrinogen, and C-reactive protein, using the Multi-Ethnic Study of Atherosclerosis (MESA). The MESA sample was free of clinical atherosclerotic disease at baseline, and thus it is well-suited for examining the relationship between social support and physiologic changes isolated from the confounding effects of pre-existing CVD that may mask true associations or create spurious ones. While alterations in these inflammatory markers may not have immediate clinical significance, they may be early indicators of cardiovascular disease risk.

We investigated two main questions: (1) Is low social support directly associated with these inflammatory markers? and (2) Does high social support buffer the relationship between stress and inflammation? We hypothesized that if buffering is predominant, the association between stress and inflammation will be stronger among those experiencing lower social support than among those experiencing higher social support. However, if the direct model is predominant, the association of chronic stress with inflammation will not vary by level of support (and the association of social support with inflammation will not vary by stress level). In addition, we investigated whether these associations varied by age and gender.

\section{Methods}

\subsection{Sample}

MESA is an on-going population-based multi-site study of the predictors of subclinical cardiovascular disease. Participants aged 45-84 years and free of clinical CVD at baseline (e.g., never experienced a heart attack, stroke, transient ischemia attack, heart failure, angina, atrial fibrillation, or cardiovascular procedures) were recruited from six study sites (Baltimore City and Baltimore County, MD; Chicago, IL; Forsyth County, NC; Los Angeles County, CA; New York City, NY; and St. Paul, MN). The final sample was 53\% female, $40 \%$ non-Hispanic white, 30\% African American, 20\% Hispanic, and $10 \%$ Asian. Details of the sampling design and study procedures have been discussed previously (Bild et al., 2002). This report is restricted to the baseline MESA sample with complete data on the measures of social support, stress, and markers of inflammation ( $N=6153,90 \%$ of the baseline sample). Participants excluded from the analysis $(N=611)$ were older than those included (mean age 66.0 vs. 61.9 years, $p<0.02$ ), but otherwise did not differ from the remaining sample in terms of race, gender, education, employment, income, marital status, body mass index (BMI), smoking status, or experience of stress (data not shown).

This study was approved by the Institutional Review Board at each site and all participants provided informed consent.

\subsection{Independent variables}

The primary independent variable was perceived emotional social support (ESS), indicated by the emotional social support index (ESSI, range: 6-30, Cronbach's alpha: 0.88 ) which consists of six 5point likert-scored items concerning availability of emotional support (e.g., Is there someone available to you whom you can count onto listen to you when you need to talk? 1 = None of the time, $2=A$ little of the time, 3 = Some of the time, $4=$ Most of the time, $5=$ All of the time), with higher values indicating more available emotional support (ENRICHD, 2000; Mitchell et al., 2003). The ESSI has modest correlations with other indices of social support (i.e., Perceived Social Support Scale) (Mitchell et al., 2003). Because exploratory analyses indicated that the relationship between social support and inflammation was non-linear, the summed scale score was categorized to indicate low (score <12), moderate (score 12-24) or high (score $\geqslant 25$ ) levels of ESS based on previous literature on social support and health in later life. In addition, each item was dichotomized as indicating low (score $\leqslant 2$ or below) or moderate/high ESS (score $\geqslant 3$ ).

Stress was measured by a composite of five items concerning contemporaneous, on-going stressors in five domains (i.e., personal health, health of a friend/relative, work-life, financial matters, and relationships with friends/relatives), with higher values indicating more stressors (Bromberger and Matthews, 1996). Each item that was endorsed was rated on a three-point scale in terms of stressfulness ( $1=$ Not very stressful, $2=$ Moderately stressful, $3=$ Very stressful) (range: $0-5$ very stressful events). The scores on this scale were strongly left-skewed, with $66 \%$ of the sample reporting only experiencing zero or one very stressful events, and therefore these responses were then combined into a dichotomous variable indicating presence of at least one very stressful event. In addition, to categorize stressors as either recent-onset or long-standing, participants were also asked if each of the stressors endorsed had been going on for 6 months or longer. If a participant responded positively to this duration question for at least on stressor, they were classified as experiencing chronic stress.

The main factors investigated as moderators of the relationship between ESS and the markers of inflammation were gender and age group, dichotomized as middle- age ( $45-64$ years, $N=3543$ ) and older adults (65-84 years, $N=2648$ ).

Additional factors included as covariates were age (centered on the sample mean, 61.9 years), sex, race/ethnicity (modeled as an indicator variable with non-Hispanic whites as the reference group), educational attainment (dichotomized as at least some college vs. high school or less (reference)), employment status (dichotomized as currently working vs. other (reference)), annual gross household income (categorized as $<\$ 20,000$ (reference), $\$ 20,000$ to $<\$ 40,000, \$ 40,000$ to $<\$ 75,000$, and $\geqslant \$ 75,000$ ), and marital status (categorized as current married (reference), divorced/separated, never married, and widowed). Three additional factors known to influence inflammatory markers, current smoking status (with former/never smoker as the reference), BMI $\left(\mathrm{kg} / \mathrm{m}^{2}\right)$, and number of alcohol drinks per week were included. Two health conditions, hypertension and diabetes, were also included. Hypertension was dichotomously indicated as systolic blood pressure $\geqslant 140 \mathrm{~mm} \mathrm{Hg}$ or diastolic $\geqslant 90 \mathrm{~mm} \mathrm{Hg}$ from the average of three resting blood pressure readings, or use of antihypertensive medications. Diabetes was determined by the American Diabetes Association 2003 fasting glucose criteria ( $\geqslant 7.0 \mathrm{mmol} / \mathrm{L}$ ) or use of insulin 
or other hypoglycemic medications (American Diabetes Association, 2006). Consistent with previous MESA reports using these inflammatory markers, recent infection status was dichotomously coded based on self-report of cold or flu, sinus infection, urinary tract infection, tooth infection, bronchitis, or pneumonia in the preceding 2 weeks and accounted for in the regression models (Ranjit et al., 2007). Use of non-steroidal anti-inflammatory drugs (NSAIDs) was also recorded.

All variables were assessed at the baseline MESA interview concurrently with the measurement of serum inflammatory markers.

\subsection{Dependent variables}

The primary outcomes were three markers of inflammation, interleukin-6 (IL-6, pg/mL), C-reactive protein (CRP, mg/L), and fibrinogen antigen $(\mathrm{mg} / \mathrm{dL})$ which have been shown to be associated with risk of cardiovascular morbidity and mortality. We conducted separate analyses with each marker as an evaluation of the robustness of the relationships to inflammation more generally. The standardized procedures for the collection, processing, shipping, and storage of blood samples have been previously described (Bild et al., 2002). Fasting venous blood samples were taken from participants at baseline and processed at a centralized laboratory. IL-6 was measured using ultrasensitive enzyme-linked immunosorbant assay (ELISA) (R\&D Systems, Minneapolis, MN). High-sensitivity CRP was measured by nephelometry (BNII nephelometer, Dade-Behring Inc., San Mateo, CA). Fibrinogen antigen was also measured by nephelometery (BNII $N$ antiserum to human fibrinogen, Dade-Behring, San Mateo, CA).

\subsection{Statistical analysis}

Initially we used multivariate analysis of variance (MANOVA) to compare mean levels of the three inflammatory markers by emotional social support and investigate interactions between ESS, gender, and age group. We used the non-parametric Wilcoxon rank-sum test for linear trend to evaluate whether covariates varied across levels of ESS (categorized as low, moderate and high, described above). We used a series of linear regression models to assess the independent association between levels of ESS and the three markers of inflammation as the outcomes. Values of the inflammatory markers were log-transformed in order to normalize their distributions to better meet the model assumptions. High ESS was the most prevalent category (55.2\% of the sample), and was used as the reference group for the analyses evaluating the direct associations between low and moderate emotional support and inflammation (Hypothesis 1). In order to evaluate whether high levels of ESS buffered the association between stress and inflammation, we dichotomized support as high vs. moderate/low, with the latter as the reference group (Hypothesis 2). We evaluated the statistical significance of the interaction between this dichotomous indicator of high ESS with the dichotomous indicator of stress (both recent onset and chronic) in order to determine whether the relationship between social support and inflammation varied by exposure to stress.

The initial multivariate regression model was adjusted for age, gender, race/ethnicity, educational attainment, income, employment status, and marital status. The second model was additionally adjusted for additional behavioral and biomedical risk factors for

Table 1

Participant characteristics by emotional social support, Multi-Ethnic Study of Atherosclerosis (2000-2002).

\begin{tabular}{|c|c|c|c|c|c|}
\hline & \multicolumn{5}{|c|}{ Level of emotional social support (ESS) } \\
\hline & Overall & Low & Moderate & High & $p$-value ${ }^{*}$ \\
\hline$N$ & 6153 & 163 & 2593 & 3397 & \\
\hline Emotional social support scale (mean, SD) & $24.2(5.23)$ & $9.1(1.6)$ & $20.3(3.4)$ & $28.0(1.8)$ & $<0.001$ \\
\hline High recent onset stress (\%) & 19.1 & 38.7 & 23.6 & 14.9 & $<0.001$ \\
\hline High chronic stress (\%) & 17.8 & 37.4 & 22.2 & 13.5 & $<0.001$ \\
\hline Age, years (mean, SD) & $61.9(10.14)$ & $62.0(9.9)$ & $61.4(10.3)$ & $62.2(10.1)$ & 0.009 \\
\hline Female (\%) & 52.4 & 50.3 & 57.3 & 48.9 & $<0.001$ \\
\hline \multicolumn{6}{|l|}{ Race (\%) } \\
\hline White & 39.3 & 35.0 & 39.8 & 39.1 & 0.156 \\
\hline Black & 27.4 & 27.6 & 26.3 & 27.9 & \\
\hline Hispanic & 21.6 & 25.8 & 20.0 & 22.8 & \\
\hline Chinese & 11.8 & 11.7 & 13.9 & 10.3 & \\
\hline \multicolumn{6}{|l|}{ Education (\%) } \\
\hline High school or less & 34.9 & 41.1 & 33.4 & 35.9 & 0.307 \\
\hline At least some college & 65.1 & 58.9 & 66.7 & 64.2 & \\
\hline \multicolumn{6}{|l|}{ Income (\%) } \\
\hline$<\$ 20,000$ & 22.4 & 35.2 & 24.7 & 20.0 & $<0.001$ \\
\hline$\$ 20,000$ to $<\$ 40,000$ & 26.7 & 37.1 & 28.8 & 24.5 & \\
\hline$\$ 40,000$ to $<\$ 75,000$ & 10.1 & 7.6 & 10.3 & 10.0 & \\
\hline$\$ 75,000+$ & 40.8 & 20.1 & 36.1 & 45.5 & \\
\hline \multicolumn{6}{|l|}{ Marital status (\%) } \\
\hline Married & 61.4 & 30.7 & 50.8 & 71.1 & $<0.001$ \\
\hline Divorced/Separated & 16.9 & 29.5 & 22.6 & 12.0 & \\
\hline Widowed & 12.6 & 18.4 & 14.6 & 10.7 & \\
\hline Never married & 9.1 & 21.5 & 11.9 & 6.3 & \\
\hline Hypertension (\%) & 44.4 & 49.1 & 42.4 & 45.5 & 0.11 \\
\hline Diabetes (\%) & 11.9 & 11.0 & 12.2 & 11.7 & 0.706 \\
\hline Alcoholic drinks per week (mean, SD) & $2.4(5.2)$ & $2.6(5.7)$ & $2.3(5.4)$ & $2.4(5.1)$ & 0.516 \\
\hline BMI, kg/m2 (mean, SD) & $28.3(5.4)$ & $28.9(5.9)$ & $28.1(5.4)$ & $28.4(05.4)$ & 0.248 \\
\hline Current smoker (\%) & 14.6 & 20.9 & 17.1 & 12.4 & $<0.001$ \\
\hline \multicolumn{6}{|l|}{ Inflammatory markers (mean, SD) } \\
\hline C-reactive protein $(\mathrm{mg} / \mathrm{L})$ & $2.4(2.2)$ & $2.7(2.1)$ & $2.4(2.2)$ & $2.4(2.2)$ & 0.125 \\
\hline Interleukin-6 (pg/mL) & $1.5(1.2)$ & $1.7(1.1)$ & $1.5(1.2)$ & $1.5(1.2)$ & 0.077 \\
\hline Fibrinogen (mg/dL) & $345.4(72.8)$ & $358.7(65.5)$ & $345.1(71.9)$ & $344.7(73.7)$ & 0.035 \\
\hline
\end{tabular}

p-value for Wilcoxon rank-sum test for linear trend across categories of ESS. 
inflammation including smoking status, alcoholic drinks per week, body mass index, hypertension, diabetes, NSAID use, and recent infection status. We also conducted post hoc sensitivity analyses by (1) adjusting for depressive symptoms as indicated by the Centers for Epidemiologic Studies Depression (CESD) scale (Radloff, 1977; Ranjit et al., 2007); (2) adjusting for self-reported arthritis; and (3) excluding participants who reported a recent infection from the analysis, in order to better account for residual effects these exposures may have on inflammation. We examined whether the direct and stress-buffering association between support and inflammation varied by age and gender in stratified analyses. Any substantial differences were tested by including appropriate interaction terms in the regression models. All analyses were conducted using STATA v.9 software (StataCorp, College Station, TX). All $p$-values refer to two-tailed tests.

\section{Results}

The sample overall reported high levels of emotional social support, and level of ESS was similar for men and women $(M=24.7(\mathrm{SD}=5.2)$ for men, $M=23.8(\mathrm{SD}=5.2)$ for women out of a possible score of 30 ). Approximately one-third of the sample reported experiencing a very stressful event, whether recent onset or chronic. Women reported substantially more recent onset $(M$ : 1.35 vs. $1.05, p<0.001)$ and chronic ( $M: 1.21$ vs. $0.95, p<0.001)$ stress than men. Lower levels of support were associated with younger age, being male, having lower household income, marital status, being a current smoker, and prevalence of both recent onset and chronic stress. The three inflammatory makers were moderately correlated with each other $\left(r^{2}=0.41-0.46\right.$, all $\left.p<0.001\right)$. Mean levels of all inflammatory markers were higher among those reporting low ESS relative to high, although this pattern was only statistically significant for fibrinogen (Table 1). Mean levels of inflammatory markers were higher in women than in men and increased with age.

MANOVA indicated no relationship between the continuous measure of emotional support and the three inflammatory markers. However, levels of the markers did vary according to the categories of low, moderate, and high levels of ESS $(F=2.42, p<0.024)$, consistent with the exploratory analysis indicating the relationship was non-linear. Interaction terms between ESS levels and gender and ESS and age were not statistically significant (data not shown).

\subsection{Evaluating the direct hypothesis}

Bivariate regression indicated that low levels of ESS were associated with elevated levels of all three inflammatory markers in the sample overall (Tables 2-4), consistent with the direct hypothesis. After adjustment for demographic and socioeconomic characteristics, low ESS remained significantly associated with elevated CRP and fibrinogen, but not IL-6 (Model 1, Tables 2-4). After additional adjustment for health behaviors and biomedical factors, only CRP remained significantly associated with low support (Model 2, Tables 2-4). Stratification by sex indicated that the association of ESS with CRP was stronger and more likely to be statistically significant in men than in women ( $p$ for interaction 0.039 in fully-adjusted model). Within each gender there was no evidence that the association between low ESS differed significantly by age for CRP, IL-6 or fibrinogen ( $p$ for interaction $>0.05$ for all markers).

We conducted sensitivity analyses to determine whether depressive symptoms, arthritis, or recent infection were strong confounders in the stress-social support relationship. When CESD score was included in the models presented in Tables 3-5 the results did not change appreciably, and the interpretation that social support is only weakly directly associated with inflammation was
Table 2

Evaluating the direct model: mean difference in log-IL-6 by categories of emotional social support.

\begin{tabular}{|c|c|c|c|}
\hline & $\begin{array}{l}\text { ESS moderate vs. } \\
\text { high B }(95 \% \mathrm{CI})\end{array}$ & $\begin{array}{l}\text { ESS low vs. high } \\
\text { B (95\% CI) }\end{array}$ & $R^{2}$ \\
\hline \multicolumn{4}{|l|}{ Entire sample } \\
\hline Unadjusted & $0.01(-0.02,0.05)$ & $0.13(0.03,0.24)^{*}$ & 0.001 \\
\hline Model 1 & $0.02(-0.02,0.05)$ & $0.09(-0.01,0.20)$ & 0.097 \\
\hline Model 2 & $0.02(-0.02,0.05)$ & $0.06(-0.04,0.15)$ & 0.230 \\
\hline \multicolumn{4}{|l|}{ Men } \\
\hline Unadjusted & $0.02(-0.03,0.07)$ & $0.15(0.01,0.30)^{*}$ & 0.002 \\
\hline Model 1 & $0.03(-0.02,0.08)$ & $0.10(-0.05,0.24)$ & 0.103 \\
\hline Model 2 & $0.02(-0.03,0.07)$ & $0.05(-0.09,0.18)$ & 0.187 \\
\hline \multicolumn{4}{|l|}{ Women } \\
\hline Unadjusted & $-0.01(-0.05,0.05)$ & $0.11(-0.03,0.26)$ & 0.001 \\
\hline Model 1 & $0.01(-0.04,0.06)$ & $0.07(-0.07,0.22)$ & 0.102 \\
\hline Model 2 & $0.02(-0.03,0.06)$ & $0.06(-0.07,0.18)$ & 0.276 \\
\hline \multicolumn{4}{|c|}{ Men, age 45-64 } \\
\hline Unadjusted & $0.02(-0.05,0.08)$ & $0.14(-0.05,0.34)$ & 0.001 \\
\hline Model 1 & $0.03(-0.04,0.10)$ & $0.08(-0.12,0.28)$ & 0.050 \\
\hline Model 2 & $0.02(-0.04,0.09)$ & $0.07(-0.12,0.26)$ & 0.152 \\
\hline \multicolumn{4}{|c|}{ Men, age $65-84$} \\
\hline Unadjusted & $0.05(-0.02,0.13)$ & $0.17(-0.04,0.37)$ & 0.003 \\
\hline Model 1 & $0.02(-0.05,0.10)$ & $0.12(-0.09,0.33)$ & 0.076 \\
\hline Model 2 & $0.01(-0.07,0.08)$ & $0.03(-0.17,0.23)$ & 0.133 \\
\hline \multicolumn{4}{|c|}{ Women, age 45-64 } \\
\hline Unadjusted & $-0.02(-0.08,0.05)$ & $0.20(0.01,0.39)^{*}$ & 0.003 \\
\hline Model 1 & $0.01(-0.06,0.06)$ & $0.13(-0.06,0.32)$ & 0.105 \\
\hline Model 2 & $0.01(-0.04,0.07)$ & $0.09(-0.07,0.26)$ & 0.300 \\
\hline \multicolumn{4}{|c|}{ Women, age 65-84 } \\
\hline Unadjusted & $0.03(-0.04,0.09)$ & $-0.01(-0.22,0.21)$ & 0.001 \\
\hline Model 1 & $0.02(-0.05,0.09)$ & $-0.02(-0.24,0.20)$ & 0.065 \\
\hline Model 2 & $0.01(-0.05,0.08)$ & $-0.02(-0.22,0.18)$ & 0.209 \\
\hline
\end{tabular}

Model 1 adjusted for age, gender, race/ethnicity, educational attainment, income, employment status, and marital status.

Model 2 adjusted for Model 1 plus smoking status, alcohol use, body mass index, hypertension, diabetes, medication use, and recent infection.

$p<0.05$. Values of IL-6 have been log-transformed.

upheld (data available upon request). Neither additional adjustment for arthritis, nor excluding participants who had experienced a recent infection $(N=1467)$, substantially influenced the results (data available upon request).

\subsection{Evaluating the buffering hypothesis}

Stress was consistently associated with elevated levels of all inflammatory markers in main effect models (data not shown). However, there was no support for the stress-buffering hypothesis in the full sample or sex-stratified analyses, either in bivariate or multivariable regression models for any of the inflammatory markers (Table 5 shows results for CRP only. The other inflammatory markers revealed similar patterns and are not shown). In age- and sex-stratified analyses, interactions terms were consistently negative, consistent with a buffering, but the magnitude of the heterogeneity was small and it was not statistically significant except in the case of 45-64 year old women. In this group, there was evidence that high ESS buffered the association between high stress and CRP in the fully-adjusted models ( $p$ for interaction 0.042). There was no evidence of buffering for the other two markers among middle-age women. Additional analyses focused on chronic stress (lasting 6 months or longer) produced similar results (data not shown).

\subsection{Sensitivity analysis: marriage as a proxy for emotional social support}

Marriage is a key source of social support, particularly for older adults, and ESS and levels of IL-6, CRP and fibrinogen varied signif- 
Table 3

Evaluating the direct model: mean difference in log-CRP by categories of emotional social support

\begin{tabular}{|c|c|c|c|}
\hline & $\begin{array}{l}\text { ESS moderate vs. } \\
\text { high } \mathrm{B}(95 \% \mathrm{CI})\end{array}$ & $\begin{array}{l}\text { ESS low vs. high } \\
\text { B }(95 \% \mathrm{CI})\end{array}$ & $\mathrm{R} 2$ \\
\hline \multicolumn{4}{|l|}{ Entire sample } \\
\hline Unadjusted & $0.01(-0.04,0.06)$ & $0.21(0.04,0.37)^{*}$ & 0.001 \\
\hline Model 1 & $0.01(-0.04,0.07)$ & $0.20(0.04,0.36)^{*}$ & 0.099 \\
\hline Model 2 & $0.02(-0.03,0.07)$ & $0.15(0.01,0.30)^{*}$ & 0.226 \\
\hline \multicolumn{4}{|l|}{ Men } \\
\hline Unadjusted & $0.02(-0.05,0.10)$ & $0.30(0.08,0.51)^{*}$ & 0.003 \\
\hline Model 1 & $0.05(-0.03,0.12)$ & $0.30(0.08,0.52)^{*}$ & 0.073 \\
\hline Model 2 & $0.03(-0.04,0.10)$ & $0.24(0.04,0.44)^{*}$ & 0.193 \\
\hline \multicolumn{4}{|l|}{ Women } \\
\hline Unadjusted & $-0.06(-0.14,0.02)$ & $0.09(-0.14,0.32)$ & 0.001 \\
\hline Model 1 & $-0.02(-0.09,0.06)$ & $0.10(-0.13,0.33)$ & 0.078 \\
\hline Model 2 & $-0.01(-0.07,0.07)$ & $0.07(-0.15,0.28)$ & 0.229 \\
\hline \multicolumn{4}{|c|}{ Men, age 45-64 } \\
\hline Unadjusted & $-0.01(-0.11,0.09)$ & $0.27(-0.03,0.57)$ & 0.002 \\
\hline Model 1 & $0.06(-0.04,0.16)$ & $0.24(-0.05,0.54)$ & 0.084 \\
\hline Model 2 & $0.04(-0.05,0.13)$ & $0.26(-0.01,0.53)$ & 0.222 \\
\hline \multicolumn{4}{|c|}{ Men, age $65-84$} \\
\hline Unadjusted & $0.08(-0.04,0.19)$ & $0.33(0.01,0.65)^{*}$ & 0.005 \\
\hline Model 1 & $0.04(-0.07,0.15)$ & $0.37(0.05,0.70)^{*}$ & 0.064 \\
\hline Model 2 & $0.02(-0.09,0.13)$ & $0.25(-0.06,0.56)$ & 0.163 \\
\hline \multicolumn{4}{|c|}{ Women, age 45-64 } \\
\hline Unadjusted & $-0.08(-0.19,0.02)$ & $0.17(-0.14,0.49)$ & 0.003 \\
\hline Model 1 & $-0.01(-0.11,0.10)$ & $0.16(-0.15,0.47)$ & 0.102 \\
\hline Model 2 & $0.03(-0.07,0.12)$ & $0.11(-0.17,0.38)$ & 0.277 \\
\hline \multicolumn{4}{|c|}{ Women, age 65-84 } \\
\hline Unadjusted & $-0.03(-0.14,0.08)$ & $-0.03(-0.37,0.32)$ & 0.001 \\
\hline Model 1 & $-0.03(-0.14,0.09)$ & $-0.01(-0.36,0.33)$ & 0.084 \\
\hline Model 2 & $-0.04(-0.15,0.07)$ & $-0.04(-0.37,0.28)$ & 0.202 \\
\hline
\end{tabular}

Model 1 adjusted for age, gender, race/ethnicity, educational attainment, income, employment status, and marital status.

Model 2 adjusted for Model 1 plus smoking status, alcohol use, body mass index, hypertension, diabetes, medication use, and recent infection.

$p<0.05$. Values of CRP have been log-transformed.

icantly by marital status (all $p<0.001$ ). We therefore conducted a series of post hoc analyses to evaluate whether marital status moderated the association between stress and inflammation. In multivariate regression analyses, the relationship between stress and inflammation did not significantly differ by marital status in the sample overall, indicated by the non-significant interaction terms between marital status and stress (data not shown). Similar results were obtained in the age- and sex-stratified analyses.

\section{Discussion}

The main finding from this study is that perceived emotional social support has little influence, either through direct or stressbuffering pathways, on inflammatory markers in this diverse sample of adults free of prevalent CVD. These findings are broadly consistent with recent reports that indicate only modest associations between perceived social support and integration with inflammation after accounting for perceived stress (McDade et al., 2006). There was modest evidence that the relationship between ESS and inflammation differed for men and women and by age. Among men, there was evidence to support the direct hypothesis of social support but only for CRP. In adjusted models there was evidence that high ESS buffered the association between high stress and CRP, but only for middle-age women. However, because these relationships were only observed with one of the three inflammatory markers examined, they should be interpreted with caution.

In fully-adjusted analyses, there was no evidence to support either the direct or buffering hypotheses for IL-6 or fibrinogen. The inconsistency in the relationships among ESS and the three
Table 4

Evaluating the direct model: mean difference in log-fibrinogen by categories of emotional social support.

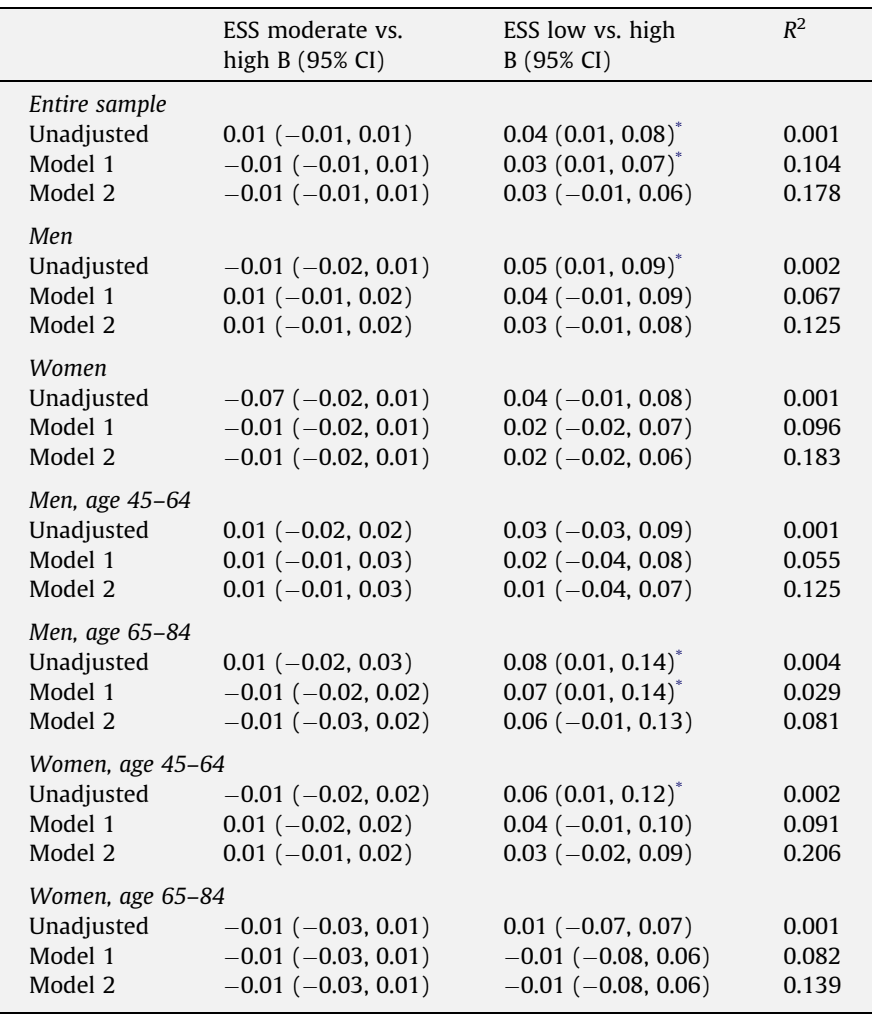

Model 1 adjusted for age, gender, race/ethnicity, educational attainment, income employment status, and marital status.

Model 2 adjusted for Model 1 plus smoking status, alcohol use, body mass index, hypertension, diabetes, medication use, and recent infection.

$p<0.05$. Values of fibrinogen have been log-transformed.

inflammatory markers is noteworthy because it suggests that the association between social support and inflammation is not equivalent across physiologic systems. These markers were only moderately correlated, and each has different responsiveness to social stressors (Ranjit et al., 2007) and may be influenced by health behaviors, particularly smoking and obesity, to differing degrees (Ozbay et al., 2008; Piché et al., 2005).

These findings are somewhat consistent with previous reports suggesting that the relationship between social support and health differs for women and men over the life span (Akiyama and Antonucci, 1996; Loucks et al., 2006a), but suggest that in adults free from major health problems such as CVD these differences are less pronounced. Several studies have reported gender differences in the associations between social integration with inflammatory markers (Ford et al., 2006; Loucks et al., 2006a,b; Loucks et al., 2006a) or measures of cardiovascular activity (Hughes, 2007). In particular, similar to our findings, several previous studies have found a direct association between measures of support or integration and inflammatory markers among men but not women. The reasons for this gender difference are unclear, although there is suggestive evidence that men and women differentially utilize social support as a coping strategy in the face of on-going stress and may appraise stress differently (Chaplin et al., 2008; Gerin et al., 1995; Unger et al., 1999). Future research should work towards identifying the specific contexts and points in the life course in which gender differences in social support and health are expected to be most relevant.

The primary strength of this study is the sample composition and measures of inflammation. The MESA sample was free of 
Table 5

Evaluating the buffering model: mean difference in log-CRP associated with emotional social support, chronic stress, and their interaction.

\begin{tabular}{|c|c|c|c|}
\hline & Unadjusted B (95\% CI) & Model 1 B (95\% CI) & Model 2 B (95\% CI) \\
\hline $\begin{array}{l}\text { Entire sample } \\
\text { High ESS (Ref. low/moderate) } \\
\text { High recent stress (Ref. low/moderate) } \\
\text { High ESS high stress }\end{array}$ & $\begin{array}{l}0.02(-0.04,0.07) \\
0.24(0.15,0.34)^{*} \\
-0.09(-0.22,0.05)\end{array}$ & $\begin{array}{l}-0.01(-0.06,0.05) \\
0.10(0.01,0.19)^{*} \\
-0.06(-0.20,0.07)\end{array}$ & $\begin{array}{l}-0.01(-0.07,0.04) \\
0.04(-0.05,0.12) \\
-0.06(-0.18,0.06)\end{array}$ \\
\hline $\begin{array}{l}\text { Men } \\
\text { High ESS (Ref. low/moderate) } \\
\text { High recent stress (Ref. low/moderate) } \\
\text { High ESS high stress }\end{array}$ & $\begin{array}{l}-0.03(-0.10,0.05) \\
0.15(-0.01,0.30) \\
-0.05(-0.26,0.17)\end{array}$ & $\begin{array}{l}-0.06(-0.14,0.02) \\
0.06(-0.09,0.21) \\
-0.02(-0.23,0.19)\end{array}$ & $\begin{array}{l}-0.04(-0.11,0.04) \\
0.02(-0.11,0.16) \\
-0.03(-0.23,0.17)\end{array}$ \\
\hline $\begin{array}{l}\text { Women } \\
\text { High ESS (Ref. low/moderate) } \\
\text { High stress (Ref. low/moderate) } \\
\text { High ESS high stress* }\end{array}$ & $\begin{array}{l}0.10(0.01,0.19) \\
0.22(0.10,0.33)^{*} \\
-0.14(-0.31,0.04)\end{array}$ & $\begin{array}{l}0.05(-0.04,0.13) \\
0.12(0.01,0.24)^{*} \\
-0.12(-0.29,0.05)\end{array}$ & $\begin{array}{l}0.03(-0.06,0.11) \\
0.06(-0.05,0.17) \\
-0.11(-0.27,0.05)\end{array}$ \\
\hline $\begin{array}{l}\text { Men, age } 45-64 \\
\text { High ESS (Ref. low/moderate) } \\
\text { High recent stress (Ref. low/moderate) } \\
\text { High ESS high stress }\end{array}$ & $\begin{array}{l}0.01(-0.10,0.12) \\
0.13(-0.05,0.32) \\
-0.09(-0.35,0.18)\end{array}$ & $\begin{array}{l}-0.06(-0.17,0.05) \\
0.01(-0.17,0.19) \\
-0.02(-0.28,0.25)\end{array}$ & $\begin{array}{l}-0.05(-0.15,0.06) \\
-0.01(-0.17,0.17) \\
-0.05(-0.29,0.19)\end{array}$ \\
\hline $\begin{array}{l}\text { Men, age } 65-84 \\
\text { High ESS (Ref. low/moderate) } \\
\text { High recent stress (Ref. low/moderate) } \\
\text { High ESS high stress }\end{array}$ & $\begin{array}{l}-0.08(-0.19,0.03) \\
0.28(0.01,0.55)^{*} \\
-0.04(-0.42,0.33)\end{array}$ & $\begin{array}{l}-0.05(-0.16,0.07) \\
0.19(-0.09,0.47) \\
-0.07(-0.45,0.30)\end{array}$ & $\begin{array}{l}-0.03(-0.14,0.08) \\
0.10(-0.17,0.36) \\
-0.03(-0.39,0.32)\end{array}$ \\
\hline $\begin{array}{l}\text { Women, age } 45-64 \\
\text { High ESS (Ref. low/moderate) } \\
\text { High recent stress (Ref. low/moderate) } \\
\text { High ESS high stress }\end{array}$ & $\begin{array}{l}0.14(0.03,0.26)^{*} \\
0.34(0.18,0.49)^{*} \\
-0.18(-0.41,0.06)\end{array}$ & $\begin{array}{l}0.05(-0.07,0.17) \\
0.25(0.10,0.40)^{*} \\
-0.15(-0.38,0.07)\end{array}$ & $\begin{array}{l}0.03(-0.07,0.14) \\
0.19(0.05,0.32)^{*} \\
-0.21(-0.42,-0.01)^{*}\end{array}$ \\
\hline $\begin{array}{l}\text { Women, age } 65-84 \\
\text { High ESS (Ref. low/moderate) } \\
\text { High stress (Ref. low/moderate) } \\
\text { High ESS high stress }\end{array}$ & $\begin{array}{l}0.05(-0.08,0.17) \\
0.03(-0.15,0.21) \\
-0.08(-0.35,0.19)\end{array}$ & $\begin{array}{l}0.03(-0.09,0.16) \\
-0.05(-0.24,0.13) \\
-0.06(-0.33,0.21)\end{array}$ & $\begin{array}{l}0.03(-0.09,0.15) \\
-0.11(-0.28,0.06) \\
0.02(-0.24,0.27)\end{array}$ \\
\hline
\end{tabular}

Model 1 adjusted for age, gender, race/ethnicity, educational attainment, income, employment status, and marital status.

Model 2 adjusted for Model 1 plus smoking status, alcohol use, body mass index, hypertension, diabetes, medication use, and recent infection.

$p<0.05$. Values of CRP have been log-transformed.

CVD at baseline, thus reducing the likelihood that poor health status confounded the relationship between inflammation and support (i.e., individuals who report low support may do so because they have limited functioning due to health problems that are themselves associated with inflammation). Data on inflammatory markers were available on over $90 \%$ of the baseline sample and samples were collected using a standardized protocol in clinical settings.

The findings should be interpreted in light of the study limitations. Foremost, this study only explored the relationship between systemic inflammation and emotional social support, and it is possible that other aspects of social life, such as caregiving and social integration, which are also associated with morbidity and mortality, are more strongly associated with these markers. For example, a growing body of evidence suggests that perceived loneliness is associated with inflammatory markers (Hawkley and Cacioppo, 2003; Steptoe et al., 2004). These contrasting findings indicate that high levels of emotional social support cannot be conceptualized or treated as equivalent to low levels of loneliness (and vice versa) in terms of the relationship to physiology, despite similarities in these constructs. Also, other measures of social support, such as instrumental (i.e., assistance with specific tasks) or provision of support may also be more relevant to inflammation. In addition, the relatively high levels of ESS reported in the sample, and the relatively low variance in the inflammatory markers may have contributed to the failure to detect significant relationships. Finally, the measure of chronic stress may not have captured all meaningful aspects of the stress process that are relevant to health (e.g., we could not examine stress appraisal or coping, nor other aspects of stress exposure including daily hassles). The measure of chronic stress may be confounded with low ESS since some of the events included in this measure referenced social relationships, a salient limitation particularly in light of the cross-sectional nature of the study.
While the finding that ESS was generally not associated with inflammation, in either the directly or stress-buffering models, is surprising in light of the consistent epidemiologic relationships between social support and heath, these results indicate that other mechanisms may underlie the relationship between social support and health (Hawkley and Cacioppo, 2003; Uchino, 2006). For example, ESS may operate on health through other pathways such as improved access to services (i.e., having supportive ties may facilitate treatment seeking), through the relationship between ESS and health-related behaviors (i.e., smoking, diet, exercise), via psychological states such as depression, or through stress-linked biological mechanisms not involving inflammation. In addition, other dimensions of social life such as integration and isolation and perceived loneliness may be stronger predictors of inflammation (Ford et al., 2006; Seeman et al., 1994) than the ESS measure we studied.

Overall our results suggest that ESS is modestly associated with levels of CRP in men and that it may buffer the effects of stress in women. Given the large number of comparisons we performed these results need to be confirmed in other large samples. Consistently with prior work, these findings illustrate the utility of examining how factors such as gender and age influence the relationship between social life and health.

\section{Conflicting interests}

All authors declare they have no conflicting interests.

\section{Acknowledgments}

This research was supported by the Robert Wood Johnson Health and Society Scholars program and the Michigan Center for Integrative Approaches to Health Disparities, P60 MD002249 
funded by the National Center on Minority Health and Health Disparities, and by contracts N01-HC-95159 through N01-HC-95169 from the National Heart, Lung, and Blood Institute. The authors thank the other investigators, the staff, and the participants of the MESA study for their valuable contributions.

\section{References}

Ajrouch, K., Blandon, A., Antonucci, T.C., 2005. Social networks among men and women: the effects of age and socioeconomic status. J. Gerontol. Ser. B Psychol. Sci. Soc. Sci. 60B, S311-S317.

Akiyama, H., Antonucci, T.C., 1996. Same-sex and cross-sex relationships. J. Gerontol. Ser. B Psychol. Sci. Soc. Sci. 51, 374-382.

American Diabetes Association, 2006. Diagnosis and classification of diabetes mellitus. Diabetes Care 29, S43-S48.

Berkman, L.F., Blumenthal, J., Burg, M., Carney, R.M., Catellier, D., Cowan, M.J., et al., 2003. Effects of treating depression and low perceived social support on clinical events after myocardial infarction: the enhancing recovery in coronary heart disease patients (ENRICHD) randomized trial. JAMA 289, 3106-3116.

Bild, D.E., Bluemke, D.A., Burke, G.L., Detrano, R., Diez Roux, A.V., Folsom, A.R., et al., 2002. Multi-Ethnic Study of Atherosclerosis: objectives and design. Am. J. Epidemiol. 156, 871-881.

Bromberger, J.T., Matthews, K.A., 1996. A longitudinal study of the effects of pessimism, trait anxiety, and life stress on depressive symptoms in middle-aged women. Psychol Aging 11, 207-213.

Brummett, B., Barefoot, J., Siegler, I., Clapp-Channing, N., Lytle, B., Bosworth, H., et al., 2001. Characteristics of socially isolated patients with coronary artery disease who are at elevated risk for mortality. Psychosom. Med. 63, 267-272.

Chaplin, T.M., Hong, K., Bergquist, K., Sinha, R., 2008. Gender differences in response to emotional stress: an assessment across subjective, behavioral, and physiological domains and relations to alcohol craving. Alcohol. Clin. Exp. Res. $32,1242-1250$

Cohen, S., Wills, T., 1985. Stress, social support, and the buffering hypothesis. Psychol. Bull. 98, 310-357.

ENRICHDInvestigators, 2000. Enhancing recovery in coronary heart disease patients (ENRICHD): study design and methods. Am. Heart J. 139, 1-9.

Ford, E.S., Loucks, E.B., Berkman, L.F., 2006. Social integration and concentrations of C-reactive protein among US adults. Ann. Epidemiol. 16, 78-84.

Frasure-Smith, N., Lesperance, F., Gravel, G., Masson, A., Juneau, M., Talajic, M., et al., 2000. Social support, depression, and mortality during the first year after myocardial infarction. Circulation 101, 1919-1924.

Gerin, W., Milner, D., Chawla, S., Pickering, T., 1995. Social support as a moderator of cardiovascular reactivity in women: a test of the direct effects and buffering hypotheses. Psychosom. Med. 57, 16-22.

Hawkley, L.C., Cacioppo, J.T., 2003. Loneliness and pathways to disease. Brain Behav. Immun. 17, 98-105.

Hughes, B., 2007. Social support in ordinary life and laboratory measures of cardiovascular reactivity: gender differences in habituation-sensitization. Ann. Behav. Med. 34, 166-176.
Knox, S.S., Uvnas-Moberg, K., 1998. Social isolation and cardiovascular disease: an atherosclerotic pathway? Psychoneuroendocrinology 23, 877-890.

Loucks, E.B., Berkman, L.F., Gruenewald, T.L., Seeman, T.E., 2006a. Relation of social integration to inflammatory marker concentrations in men and women 70-79 years. Am. J. Cardiol. 97, 1010-1016.

Loucks, E.B., Sullivan, L.M., D’Agostino, R.B., Larson, M.G., Berkman, L.F., Benjamin, E.J., 2006b. Social networks and inflammatory markers in the Framingham heart study. J. Biosoc. Sci. 38, 835-842.

McDade, T.W., Hawkley, L.C., Cacioppo, J.T., 2006. Psychosocial and behavioral predictors of inflammation in middle-aged and older adults: the Chicago health aging, and social relations study. Psychosom. Med. 68, 376-381.

Mitchell, P.H. Powell, L., Blumenthal, J., Norten, J., Ironson, G., Pitula, C.R, et al. 2003. A short social support measure for patients recovering from myocardial infarction: the ENRICHD social support inventory. J. Cardiopulm. Rehabil. 23 398-403.

Moen, P., 2001. The gendered life course. In: Binstock, R.H., George, L.K. (Eds.), Handbook of Aging: Social Sciences. Academic Press, San Diego CA, pp. 176196.

Ozbay, F., Fitterling, H., Charney, D., Southwick, S., 2008. Social support and resilience to stress across the life span: a neurobiologic framework. Curr. Psychiatry Rep. 10, 304-310.

Piché, M.E., Lemieux, S., Weisnagel, S.J., Corneau, L., Nadeau, A., Bergeron, J., 2005. Relation of high-sensitivity C-reactive protein, interleukin-6, tumor necrosis factor-alpha, and fibrinogen to abdominal adipose tissue, blood pressure, and cholesterol and triglyceride levels in healthy postmenopausal women. Am. J. Cardiol. 96, 92-97.

Radloff, L., 1977. The CES-D scale: a self-report depression scale for research in the general population. Appl. Psychol. Meas. 1, 385-401.

Ranjit, N., Diez Roux, A.V., Shea, S., Cushman, M., Seeman, T., Jackson, S., et al., 2007. Psychosocial factors and inflammation in the multi-ethnic study of atherosclerosis. Arch. Intern. Med. 167, 174-181.

Seeman, T., Berkman, L.F., Blazer, D., Rowe, J., 1994. Social ties and support and neuroendocrine function: the MacArthur studies of successful aging. Ann. Behav. Med. 16, 95-106.

Seeman, T.E., Crimmins, E., 2001. Social environment effects on health and aging; integrating epidemiologic and demographic approaches and perspectives. Ann NY Acad. Sci. 954, 88-117.

Steptoe, A., Owen, N., Kunz-Ebrecht, S.R., Brydon, L., 2004. Loneliness and neuroendocrine, cardiovascular, and inflammatory stress responses in middleaged men and women. Psychoneuroendocrinology 29, 593-611.

Uchino, B., 2006. Social support and health: a review of physiological processes potentially underlying links to disease outcomes. J. Behav. Med. 29, 377-387.

Uchino, B.N., Holt-Lunstad, J., Uno, D., Betancourt, R., Garvey, T.S., 1999. Socia support and age-related differences in cardiovascular function: an examination of potential mediators. Ann. Behav. Med. 21 (2), 135-142.

Unger, J.B., McAvay, G., Bruce, M.L., Berkman, L.F., Seeman, T., 1999. Variation in the impact of social network characteristics on physical functioning in elderly persons: MacArthur studies of successful aging. J. Gerontol. Ser. B Psychol. Sci. Soc. Sci. 54B, S245-S251. 